\title{
Zc3h I2d, a Novel of Hypomethylated and Immune-Related for Prognostic Marker of Lung Adenocarcinoma
}

\author{
Bo Yang, ${ }^{1-3, *}$ Lin-Lin Ji, (D) ${ }^{1,2, *}$

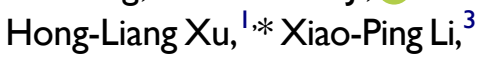 \\ Hong-Gang Zhou, ${ }^{4}$ Ting Xiao, ${ }^{4}$ \\ Xiao-He Li, ${ }^{4}$ Zhou-Yong Gao, ${ }^{1,2}$ \\ Jian-Zhong Li, ${ }^{5}$ Wei-Dong \\ Zhang,' Guang-Shun Wang, ' \\ Ming-Jiang $\mathrm{Li}^{3}$ \\ 'Department of Thoracic Surgery, Tianjin \\ Baodi Hospital, Baodi Clinical College of \\ Tianjin Medical University, Tianjin, \\ 301800, People's Republic of China; \\ ${ }^{2}$ State Key Laboratory of Proteomics, \\ Beijing Proteome Research Center, \\ National Center for Protein Sciences \\ (Beijing), Beijing, 102206, People's \\ Republic of China; ${ }^{3}$ Department of \\ Thoracic Surgery, Tianjin First Central \\ Hospital, School of Medicine, Nankai \\ University, Tianjin, 300192, People's \\ Republic of China; ${ }^{4}$ State Key Laboratory \\ of Medicinal Chemical Biology, College of \\ Pharmacy and Tianjin Key, Laboratory of \\ Molecular Drug Research, Nankai \\ University, Tianjin, 300353, People's \\ Republic of China; ${ }^{5}$ Department of \\ Thoracic Surgery, The Second Affiliated \\ Hospital of Xi'an Jiaotong University, \\ Xi'an, 710004, People's Republic of China
}

*These authors contributed equally to this work

Correspondence: Guang-Shun Wang Department of Thoracic Surgery, Tianjin Baodi Hospital, Baodi Clinical College of Tianjin Medical University, Tianjin, 301800, People's Republic of China Email wgs@bddhospital.com

Ming-Jiang Li

Department of Thoracic Surgery, Tianjin First Central Hospital, School of Medicine, Nankai University, Tianjin, 300192, People's Republic of China Email mingjiangli@nankai.edu.cn
Background: Zc3h12d is a negative regulator which plays a crucial role in immune modulation. However, the role of $\mathrm{zc} 3 \mathrm{~h} 12 \mathrm{~d}$ in lung adenocarcinoma (LUAD) remains unclear. We aim to explore the prognostic of $z c 3 h 12 d$ and investigate the relationship between zc3h12d expression and immune infiltration in LUAD.

Methods: TIMER site was used to analyze the expression of $\mathrm{zc} 3 \mathrm{~h} 12 \mathrm{~d}$ in LUAD. The zc3h12d protein levels in patient tissue samples were detected by immunohistochemistry staining assays. Meanwhile, based on UALCAN database and samples' data from our cohort, we explored the relationship of clinicopathological features and $\mathrm{zc} 3 \mathrm{~h} 12 \mathrm{~d}$ expression to determine the clinical effect of $\mathrm{zc} 3 \mathrm{~h} 12 \mathrm{~d}$ in LUAD. Several databases including GEPIA, Kaplan-Meier plotter and our samples' data were used to explore the prognostic value of zc3h12d in LUAD. Cox regression analysis was established to further evaluate the prognostic value of zc3h12d in LUAD. In addition, zc3h12d promoter methylation was analyzed by UALCAN database. Genetic alteration analysis was observed in the cBioPortal web. GO and KEGG analyses were conducted to elucidate the underlying mechanisms. Finally, the correlation between zc3h12d and tumor-infiltrating immune cells in LUAD was investigated by TIMER database. The B cells level was investigated by flow cytometry analysis of peripheral blood from our LUAD cohort.

Results: Zc3h12d expression was significantly higher in LUAD, compared with adjacent normal tissues. The clinical data from the UALCAN database demonstrated that $\mathrm{zc} 3 \mathrm{~h} 12 \mathrm{~d}$ expression was closely related with cancer stage and nodal metastasis. However, patient sample detection revealed that $\mathrm{zc} 3 \mathrm{~h} 12 \mathrm{~d}$ expression was closely related to pathological $\mathrm{N}(\mathrm{p}=$ $0.0431)$ and grade $(\mathrm{p}=0.004)$. Moreover, low $\mathrm{zc} 3 \mathrm{~h} 12 \mathrm{~d}$ expression was associated with poorer overall survival in LUAD. We analyzed the methylation level of zc3h12d in LUAD and found that the methylation levels of $\mathrm{zc} 3 \mathrm{~h} 12 \mathrm{~d}$ promoter in LUAD were significantly reduced. In addition, zc3h12d genetic alterations, including deep deletion, could be found in LUAD. GO and KEGG pathway analysis results indicated that $\mathrm{zc} 3 \mathrm{~h} 12 \mathrm{~d}$ has a certain value in immune infiltration. We investigated the expression of $z c 3 h 12 \mathrm{~d}$ in tumor-immune interactions. It was found that $\mathrm{zc} 3 \mathrm{~h} 12 \mathrm{~d}$ might be associated with the immune infiltration and markers of infiltrating immune cells of LUAD. The results of patient sample detection confirmed that B cells level was significantly lower in the patients with low zc3h12d expression than those in the patients with high zc3h12d expression.

Conclusion: zc3h12d might be considered as a potential biomarker for determining prognosis and immune-related therapeutic target in LUAD.

Keywords: zc3h12d, lung adenocarcinoma, hypomethylation, tumor microenvironment, prognosis 


\section{Introduction}

Although surgical resection, chemotherapy, radiotherapy, targeted therapy, and immunotherapy show responses during the treatment of non-small cell lung cancer (NSCLC), the survival rate of the patients remains poor in the $21 \mathrm{st}$ century. ${ }^{1}$ Lung adenocarcinoma (LUAD) represented the most frequent types of NSCLC, which account for up to $40 \%$ of all lung cancer cases. ${ }^{2}$ It is noteworthy that DNA methylation was shown to correlate with the progression of LUAD. ${ }^{3}$ Recent studies also have shed light on the diverse immune cell infiltration for LUAD. ${ }^{4}$ Under this background, new prognostic markers and incorporating of methylation and immune information are urgent needs for NSCLC prognostication.

The CCCH-zinc finger protein family consisted of four members: zc3h12a, zc3h12b, zc3h12c and zc3h12d. ${ }^{5}$ A previous study suggested that $\mathrm{CCCH}$ zinc finger motifs bind to DNA, RNA, and proteins. ${ }^{6} \mathrm{Zc} 3 \mathrm{~h} 12 \mathrm{~d}$ was enriched in spleen, lung, and lymph node. ${ }^{7}$ Researches showed that zc3h12d exerted important functions in immune modulation and inflammation. Several studies revealed that $\mathrm{zc} 3 \mathrm{~h} 12 \mathrm{~d}$ attenuated the inflammatory factors by $\mathrm{T}$ lymphocytes. ${ }^{8}$ In addition, overexpression of zc3h12d significantly inhibited TLRinduced activation of JNK, ERK and NF- $\mathrm{kB}$ in macrophages. ${ }^{9}$

As a novel tumor suppressor gene p34 in lung cancer, zc3h12d was revealed previously, but the association of patients with LUAD remains unknown. Therefore, the goal of this study was to determine the clinical usefulness of zc3h12d in LUAD patients.

\section{Materials and Methods}

Tissue and Immunohistochemistry (IHC)

All tumor specimens with LUAD after surgical resection were collected between January 2018 and December 2019 in this cohort study. None of the patients received chemotherapy, radiotherapy, and/or immunotherapy prior to resection. This study was performed in accordance with the Declaration of Helsinki and was approved by the Committees for Ethical Review of Research involving human subjects at the Tianjin First Central Hospital, School of Medicine, Nankai University (Tianjin, China; approval no. 2018N054KY). Written informed consent was obtained from all participants. The demographic and clinical characteristics of patients are listed in Table 1. All IHC staining was performed as described in the previous study. ${ }^{10}$ Staining percentage of zc3h12d was categorized as follows: $0(0 \%), 1(0-10 \%), 2$ (11-50\%), $3(51-70 \%)$, and $4(\geq 71 \%)$ and the staining intensity was stratified as follows: none $(-, 0)$, faint $(+, 1$, yellow staining), moderate $(++, 2$, light brown staining), or strong $(+++, 3$, dark brown staining). Based on the IHC score of 0 to 7 (staining percentage + intensity), zc3h12d in IHC tissue was evaluated and divided into low groups ( $<3$ points) and high groups ( $4-7$ points).

\section{Differential Expression Analysis}

The expression difference of $\mathrm{zc} 3 \mathrm{~h} 12 \mathrm{~d}$ in various types of tumors was observed by employing TIMER database (https://cistrome.shinyapps.io/timer/). ${ }^{11}$ Then, zch3h12d expression in LUAD patients with different clinical features in UALCAN tool was obtained (http://ualcan.path. uab.edu/analysis.html). ${ }^{12}$

\section{Survival Analysis}

The GEPIA (http://gepia.cancer-pku.cn/) and KaplanMeier (K-M) Plotter database (http://kmplot.com/analy sis/index.php? $p=$ service) platform were used to analyze the prognostic value of $\mathrm{zc} 3 \mathrm{~h} 12 \mathrm{~d} .{ }^{13,14}$ The cutoff value was used as the expression thresholds to divide the patients into the high- and low-zc $3 \mathrm{~h} 12 \mathrm{~d}$ cohorts.

\section{Methylation Analysis}

To evaluate association between $\mathrm{zc} 3 \mathrm{~h} 12 \mathrm{~d}$ expression and methylation levels, the promoter methylation in LUAD with different conditions was analyzed in the UALCAN database. ${ }^{12}$ And the DNA methylation sites of zc3h12d in TCGA were investigated via the MethSurv platform (https://biit.cs.ut.ee/methsurv/). ${ }^{15}$

\section{Genetic Alteration Analysis}

The results of the mutations and copy number alterations (CNAs) of the zc3h12d from all TCGA tumors were determined in the cBioPortal web (https://www.cbiopor tal.org/). ${ }^{16}$ The overall survival (OS), disease-free survival (DFS), progression-free survival (PFS), and diseasespecific survival (DSS) differences for the TCGA datasets with or without $\mathrm{zc} 3 \mathrm{~h} 12 \mathrm{~d}$ genetic alteration in LUAD patients were also obtained.

\section{Zc3hI2d-Related Gene Set Enrichment Analysis}

Furthermore, UALCAN database was used to select the relate gene set of zc3h12d. ${ }^{12}$ The biological processes (BP), cellular components (CC), molecular function (MF) and 
Table I Relationship Between zc3hI2d Expression and Clinicopathology in the LUAD Cohort

\begin{tabular}{|c|c|c|c|c|}
\hline \multirow[t]{2}{*}{ Variable } & \multirow[t]{2}{*}{ Number $(\%)(n=87)$} & \multicolumn{2}{|c|}{ zc3h/2d Expression } & \multirow[t]{2}{*}{ P-value } \\
\hline & & High (\%) (n=35) & Low (\%) (n=52) & \\
\hline \multicolumn{5}{|c|}{ Age, median (range) (years) } \\
\hline$\geq 60$ & $67(77.01)$ & $29(43.28)$ & $38(56.72)$ & 0.288 \\
\hline$<60$ & 20 (22.99) & $6(30.00)$ & I4(70.00) & \\
\hline \multicolumn{5}{|l|}{ Gender } \\
\hline Male & $50(57.47)$ & $18(36.00)$ & $32(64.00)$ & 0.350 \\
\hline Female & $37(42.53)$ & I7(45.95) & $20(54.05)$ & \\
\hline \multicolumn{5}{|l|}{ Smoking status } \\
\hline No smoking history & $4 I(47.13)$ & $19(46.34)$ & $22(53.66)$ & 0.272 \\
\hline Smoking history & $46(52.87)$ & $16(34.78)$ & $30(65.22)$ & \\
\hline \multicolumn{5}{|l|}{ PT stage } \\
\hline $\mathrm{TI} / \mathrm{T} 2$ & $80(91.95)$ & $33(41.25)$ & $47(58.75)$ & 0.799 \\
\hline $\mathrm{T} 3 / \mathrm{T} 4$ & $7(8.05)$ & $2(28.57)$ & $5(71.43)$ & \\
\hline \multicolumn{5}{|l|}{$\mathrm{pN}$ stage } \\
\hline $\mathrm{Nx} / \mathrm{N} 0 / \mathrm{NI}$ & $76(87.36)$ & $27(35.53)$ & $49(64.47)$ & $0.043 I^{*}$ \\
\hline N2/N3 & II (12.64) & $8(72.73)$ & $3(27.27)$ & \\
\hline \multicolumn{5}{|l|}{ PTNM 8th edition } \\
\hline Stage I/Stage II & $75(86.21)$ & $27(36.00)$ & $48(64.00)$ & 0.090 \\
\hline Stage III & $12(13.79)$ & $8(66.67)$ & $4(33.33)$ & \\
\hline \multicolumn{5}{|l|}{ Pathological grade } \\
\hline Grade I/Grade 2 & $64(73.56)$ & $20(31.25)$ & $44(68.75)$ & $0.004^{*}$ \\
\hline Grade 3 & $23(26.44)$ & $15(65.22)$ & $8(34.78)$ & \\
\hline
\end{tabular}

Note: *Indicates $\mathrm{p}<0.05$.

Abbreviations: $\mathrm{T}$, tumor; $\mathrm{N}$, lymph node; $\mathrm{M}$, metastasis.

KEGG pathway were visualized using the Metascape database (http://metascape.org/gp/index.html\#/main/step1) ${ }^{17}$

\section{Immune Infiltration Analysis}

To reveal the immune infiltration of zc3h12d in cancer, the correlation between zc3h12d and the immune infiltrates across all TCGA tumors were estimated by the TIMER database. ${ }^{11}$ Then, the associations of zc $3 \mathrm{~h} 12 \mathrm{~d}$ and mentioned immune cell infiltration gene markers in LUAD were explored.

\section{Measurement of B Cells}

Blood samples were collected before treatment initiation. B cells level was examined by flow cytometry analysis of peripheral blood from our LUAD cohort.

\section{Statistical Analysis}

Paired $t$-test and unpaired $t$-test were used to analyze the expression of zc3h12d in different groups. Distinctions between zc3h12d expression and clinicopathologic characteristics were evaluated by $\chi 2$ test or Fisher's exact test. The survival curves of patient were evaluated by the Log rank test. A Cox regression model was applied for the univariate and multivariate analyses of survival. Correlations of zc3h12d with immune infiltration and type markers of immune cells were analyzed using Spearman correlation. The differences in mean values between the groups were analyzed using the Mann-Whitney $U$-test. P-value $<0.05$ was considered statistically significant. All analyses and graphics were analyzed using SPSS ver. 26.0 (IBM, USA) and GraphPad Prism 8.0 (GraphPad Software, USA).

\section{Results}

\section{Zc3hI2d is Upregulated in LUAD and Closely Correlated with Clinical Characteristics}

The expression of zc3h12d in different tumor tissues was detected by the TIMER database. As shown in Figure 1A, the zc3h12d expression was significantly higher in breast invasive carcinoma (BRCA), cervical and endocervical cancer 


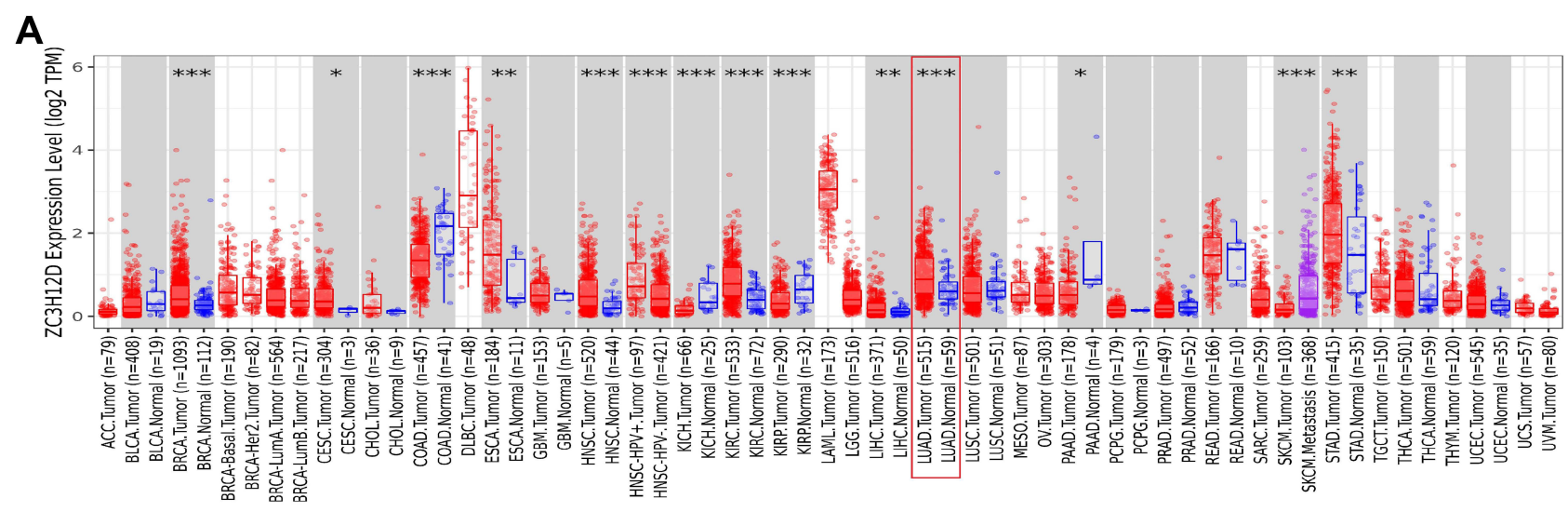

B
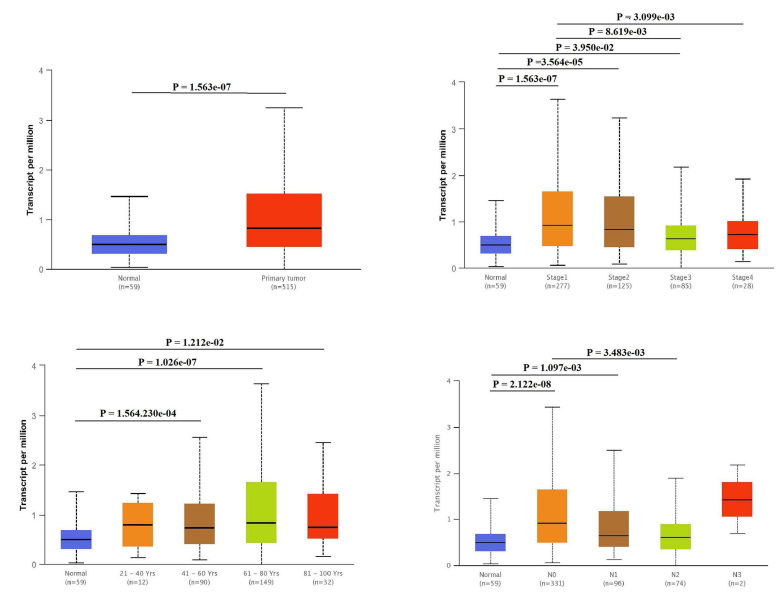
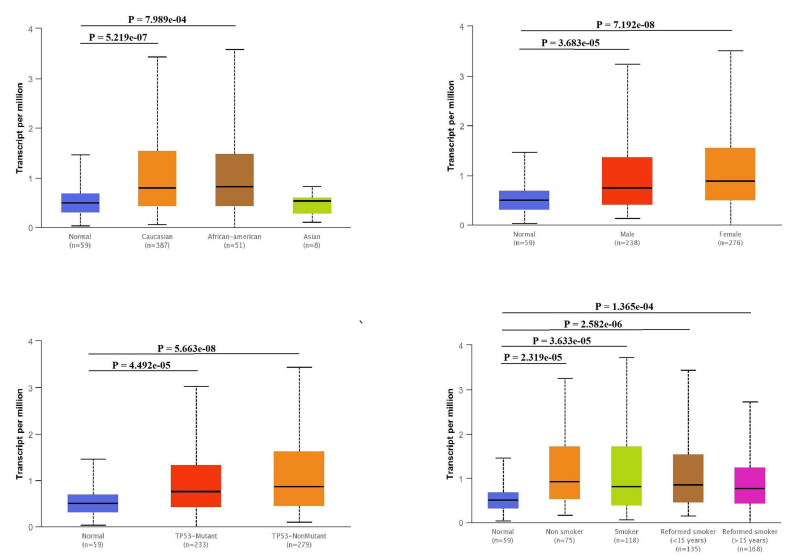

Figure I zc3h I 2d expression in LUAD. (A) zc3h I 2d expression level in different cancer types in the TCGA database. (B) zc3h I 2d expression in subtype of human LUAD. *Indicates $p<0.05$, **Indicates $p<0.01$, *** Indicates $p<0.001$.

(CESC), esophageal carcinoma (ESCA), head and neck cancer (HNSC), kidney renal clear cell carcinoma (KIRC), liver hepatocellular carcinoma (LIHC), lung adenocarcinoma (LUAD), and stomach adenocarcinoma (STAD) compared with adjacent normal tissues. However, lower expression was observed in colon adenocarcinoma (COAD), kidney chromophobe (KICH), kidney renal papillary cell carcinoma (KIRP), and pancreatic adenocarcinoma (PAAD) compared with the corresponding control tissues.

Next, the correlations between $\mathrm{zc} 3 \mathrm{~h} 12 \mathrm{~d}$ and clinical features of LUAD patients were examined. As shown in Figure $1 \mathrm{~B}$, the expression of zc3h12d was much higher in LUAD tissues than in normal tissues. Moreover, zc3h12d expression was closely related with cancer stage and nodal metastasis. Compared to the normal tissue, zc $3 \mathrm{~h} 12 \mathrm{~d}$ expression was augmented regardless of race, gender, aged (41-60, 61-80, and 81-100), and TP53 mutation status.

To further examine zc3h12d protein expression in LUAD tissues, the IHC was used to assess the zc3h12d protein expression. Results showed that zc3h12d was predominantly localized in cytoplasm. The expression of zc3h12d was significantly higher in LUAD compared to the uninvolved tissues (Figure 2A).

In addition, to validate the association between zc3h12d protein expression and clinical variables, tumor specimens were analyzed. It was found that $\mathrm{zc} 3 \mathrm{~h} 12 \mathrm{~d}$ expression was closely related to pathological $\mathrm{N}(\mathrm{p}=$ $0.0431)$ and grade $(p=0.004)$. In comparison, other clinical items, such as age $(p=0.288)$, gender $(p=0.350)$, smoking status $(\mathrm{p}=0.272), \mathrm{T}(\mathrm{p}=0.799)$ and TNM stage $(p=0.090)$ had no association with zc3h12d expression.

\section{Decreased Zc3hl2d Expression is}

\section{a Predictor of Poor Prognosis in LUAD}

The prognostic value of zc $3 \mathrm{~h} 12 \mathrm{~d}$ expression in LUAD was investigated by GEPIA analysis. Results showed that OS was significantly positively correlated with zc3h12d expression in LUAD patients (hazard ratio $[\mathrm{HR}]=0.52, \log$-rank $\mathrm{p}=2 \mathrm{e}-$ $05)$. However, the DFS in relation to zc3h12d expression was not significant $(H R=0.75, \log$-rank $p=0.066)($ Figure $2 B)$. 
A

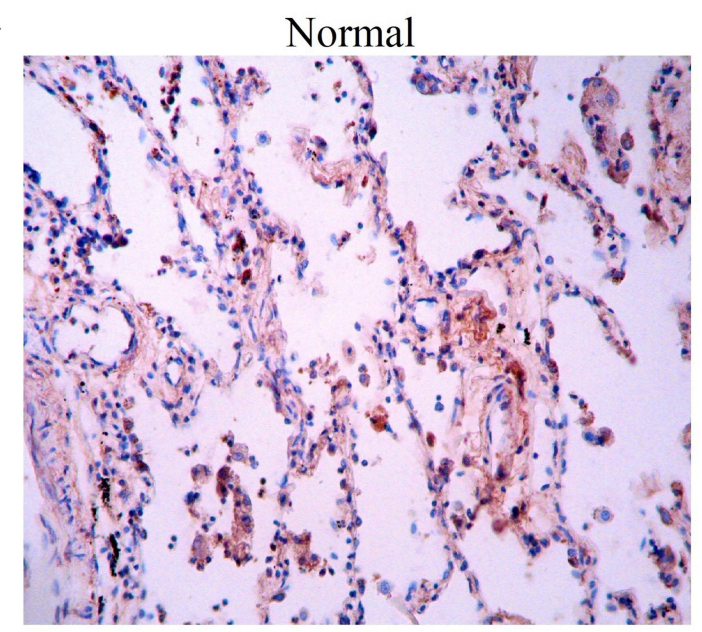

B

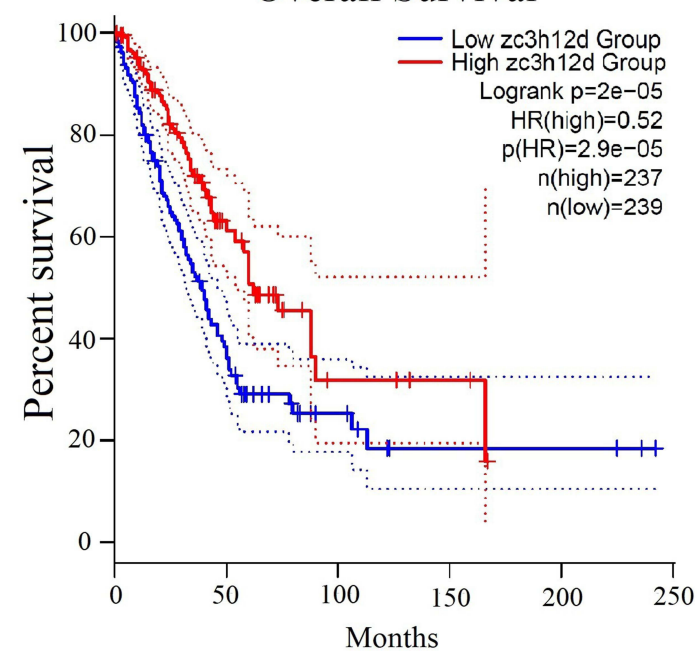

C

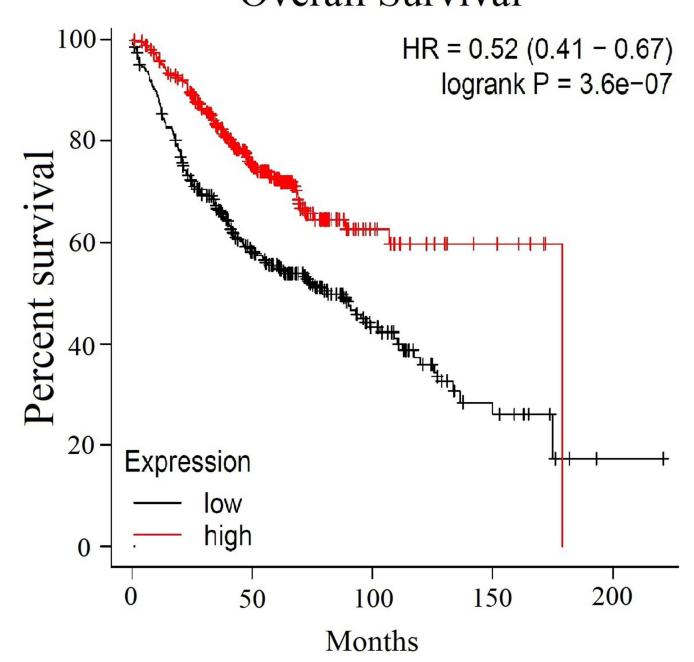

Adenocarcinoma

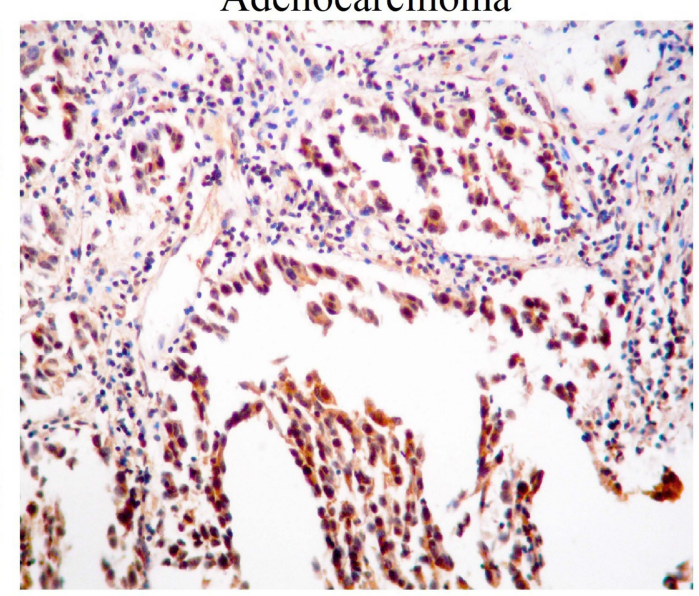

Disease Free Survival

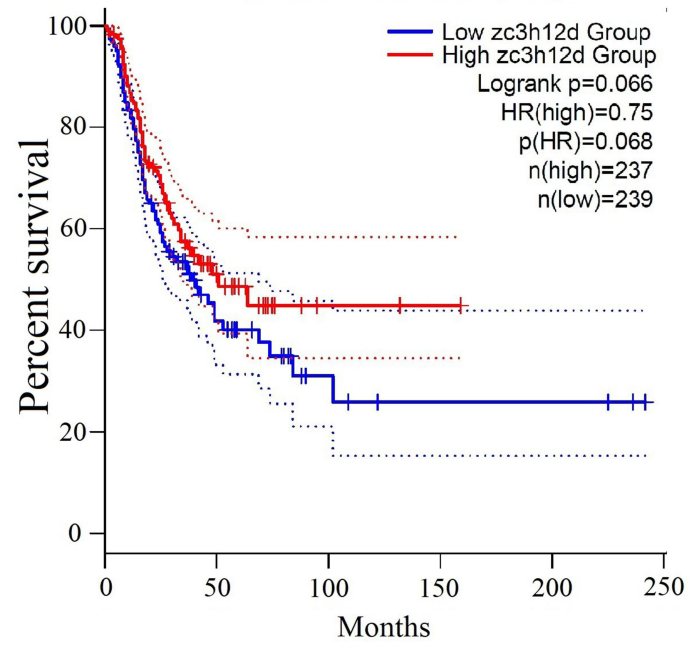

D Overall Survival

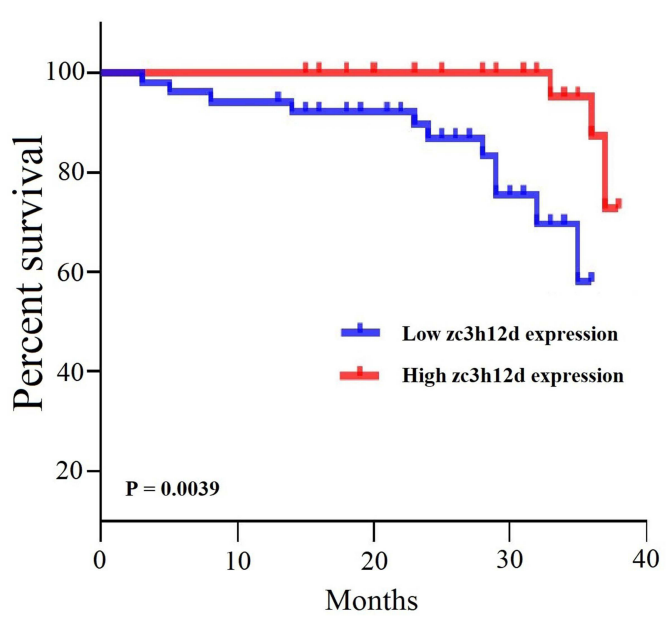

Figure $2 \mathrm{IHC}$ and prognosis of zc3h/2d in LUAD. (A) zc3h $12 \mathrm{~d}$ protein expression in LUAD tissues (200xmagnification). (B) K-M survival curves for OS and DFS in LUAD patients by GEPIA. (C) K-M survival curves for OS in LUAD patients by Kaplan-Meier Plotter database. (D) K-M survival curves of OS in LUAD patients based on tumor specimens $(n=87)$. 
Kaplan-Meier plotter database showed that high zc3h12d expression was also associated with better prognosis of OS in LUAD $(\mathrm{HR}=0.52$, log-rank $\mathrm{p}=3.6 \mathrm{e}-07$ ) (Figure $2 \mathrm{C}$ ). Moreover, analysis of prognostic significance of tumor specimens $(n=87)$ revealed that patients with low $\mathrm{zc} 3 \mathrm{~h} 12 \mathrm{~d}$ expression had shortened OS in LUAD $(\mathrm{p}=0.0039)$ (Figure 2D).

Cox regression model was performed to evaluate the prognostic potential of $\mathrm{zc} 3 \mathrm{~h} 12 \mathrm{~d}$ expression. Zc3h12d expression was a significant predictor of OS in univariate analysis $(\mathrm{HR}=7.140,95 \% \mathrm{CI} 1.542-33.064, \mathrm{p}=0.012)$ and multivariate analysis $(\mathrm{HR}=12.845,95 \% \mathrm{CI} 2.093-$ 78.828, $\mathrm{p}=0.006$ ) (Table 2).

\section{DNA Methylation Analysis Data}

The heat map of DNA methylation levels of $\mathrm{zc} 3 \mathrm{~h} 12 \mathrm{~d}$ in LUAD were analyzed and displayed in Figure 3A. Furthermore, compared with normal tissues, the methylation levels of zc3h12d promoter in LUAD was significantly reduced. We observed that zc3h12d promoter methylation levels of the cancer stage, race, gender, age, smoking status, nodal metastasis, and TP53 mutation status were lower than normal in LUAD (Figure 3B).

\section{Genetic Alteration Analysis Data}

As shown in Figure 4A, there was the highest genetic alteration of zc3h12d (8.33\%) in diffuse large B-cell lymphoma (DLBC). It was noteworthy that all LUAD cases with genetic alteration $(0.53 \%)$ had copy number deletion of $\mathrm{zc} 3 \mathrm{~h} 12 \mathrm{~d}$. In addition, the analysis of the prognostic value of LUAD patients between the zc3h12d altered group and the unaltered group showed better prognosis in disease-free $(\mathrm{p}=4.506 \mathrm{e}-06)$ and progression-free $(\mathrm{p}=1.950 \mathrm{e}-05)$ survival, but not overall $(\mathrm{p}=0.272)$ and disease-specific $(p=0.396)$ survival (Figure 4B).

\section{$\mathrm{Zc} 3 \mathrm{hl} 2 \mathrm{~d}$ is Involved in Immune Activation and Proliferation Inhibition in LUAD}

Obtain the potential zc3h12d-related gene set by UALCAN database. A total of 931 genes was finally obtained (Supplementary Table 1). GO analysis results indicated that BP category of target genes were significantly enriched in "lymphocyte activation", "adaptive immune response", and "immune response-regulating signaling pathway". CC category were mainly enriched in "side of membrane", "immunological synapse", and "plasma membrane protein complex". MF category of target genes were mainly related to "cytokine receptor activity", "GTPase binding", and "nonmembrane spanning protein tyrosine kinase activity". KEGG pathway analysis revealed that the target genes were mainly associated with "Th1 and Th2 cell differentiation", "chemokine signaling pathway", "primary immunodeficiency", "T cell receptor signaling pathway", "B cell receptor signaling pathway", and "NF-kappa B signaling pathway" (Figure 5). Based on this perspective, the zc3h12d in LUAD patients is deemed important.

\section{Relationship Between Zc3h I2d and} Tumor-Infiltrating Immune Cells in LUAD Tumor-infiltrating lymphocytes were an independent predictor of immune surveillance in determining the survival in various cancers. Therefore, we investigated whether zc3h12d expression was correlated with immune infiltration levels in LUAD. The results showed that zc3h12d was

Table 2 Univariate and Multivariate Analyses of Factors Associated with OS in LUADs Using Cox Regression

\begin{tabular}{|l|l|l|l|l|}
\hline \multirow{2}{*}{ Variable } & \multicolumn{2}{l|}{ Univariate Analysis } & \multicolumn{2}{l|}{ Multivariate Analysis } \\
\cline { 2 - 5 } & HR $(95 \%$ CI) & P-value & HR (95\% CI) & P-value \\
\hline Age $(\geq 60$ vs $<60$ years) & $0.997(0.272-3.659)$ & 0.996 & $2.965(0.578-15.204)$ & 0.193 \\
\hline Gender (Male vs Female) & $2.063(0.645-6.598)$ & 0.222 & $1.644(0.422-6.406)$ & 0.474 \\
\hline Smoking status (No smoking history vs Smoking history) & $2.019(0.668-6.095)$ & 0.213 & $0.921(0.25 I-3.38 I)$ & 0.901 \\
\hline PTNM stage (Stage Ivs. Stage 2 vs Stage3) & $1.74 I(0.965-3.139)$ & $0.035^{*}$ & $4.080(I .327-12.542)$ & $0.014^{*}$ \\
\hline Pathological grade (Grade Ivs. Grade 2 vs Grade 3) & $0.845(0.439-1.627)$ & 0.614 & $0.550(0.183-1.656)$ & 0.288 \\
\hline Zc3hI2d expression (High vs Low) & $7.140(1.542-33.064)$ & $0.012^{*}$ & $12.845(2.093-78.828)$ & $0.006 *$ \\
\hline
\end{tabular}

Note: *Indicates $\mathrm{p}<0.05$.

Abbreviations: $\mathrm{HR}$, hazard ratio; $95 \% \mathrm{Cl}, 95 \%$ confidence interval; T, tumor; $\mathrm{N}$, lymph node; M, metastasis. 
A

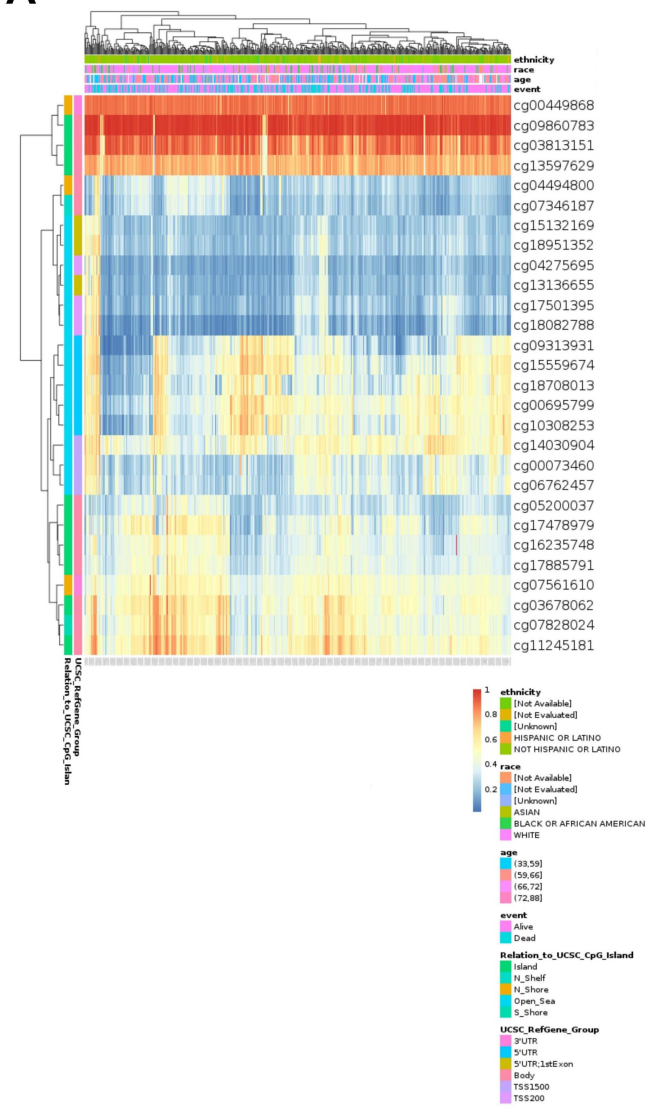

B
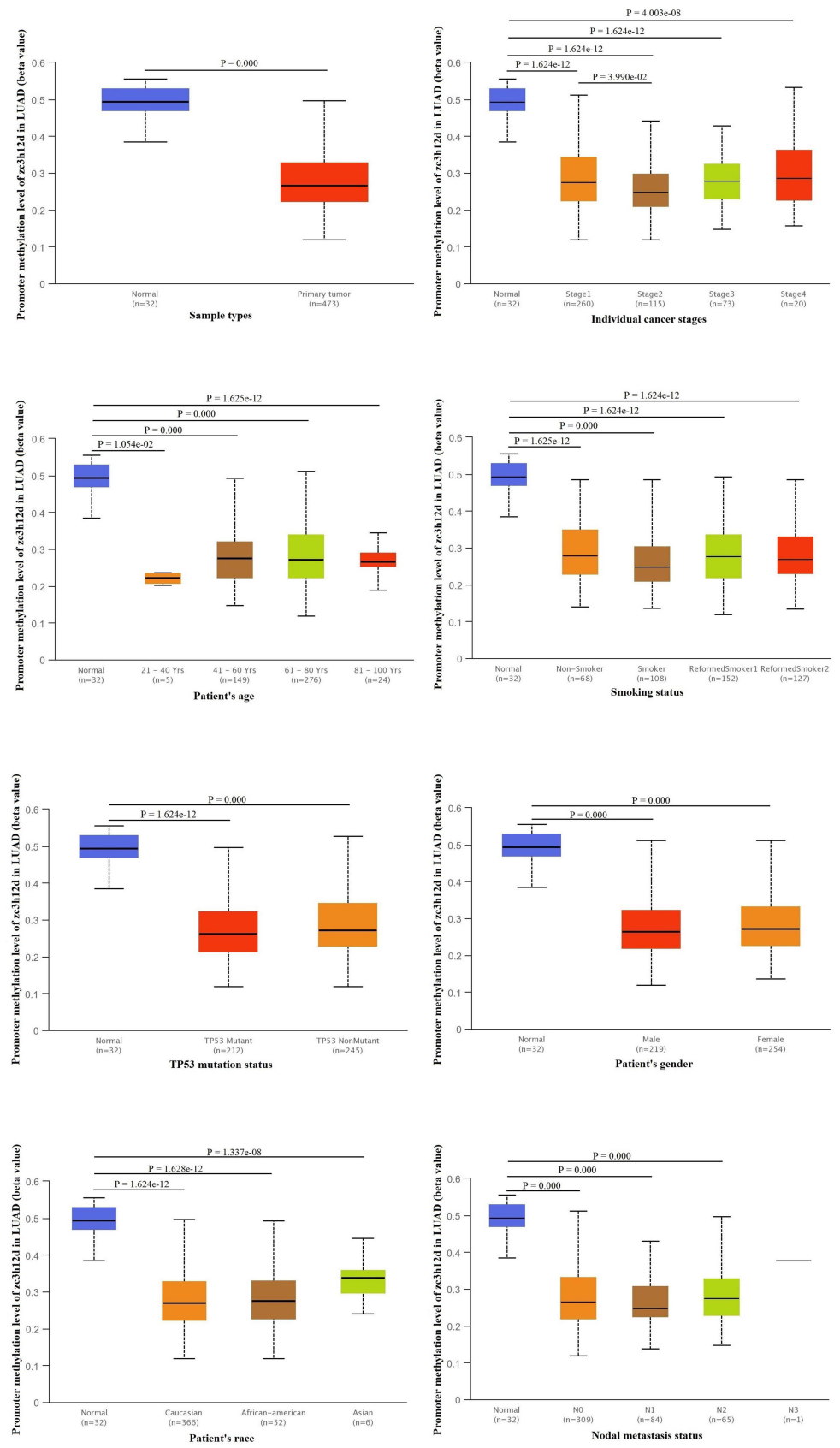

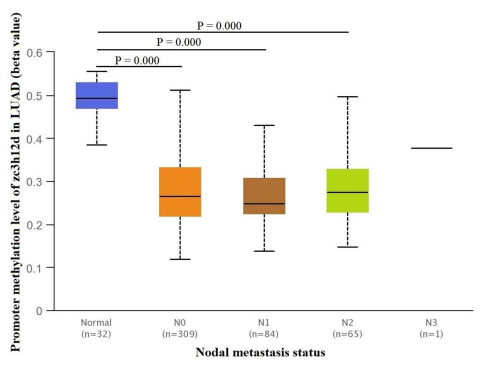

Figure 3 Methylation of $z c 3 h \mid 2 d$ in patients with LUAD. (A) The heat map of DNA methylation clustered expression of zc3h $12 \mathrm{~d}$ in LUAD. Red to blue scale indicates high to low expression. Various colorful side boxes were used to characterize the ethnicity, race, age, event, and relation to UCSC_CPG_island and UCSC_refGene_Group. (B) Promoter methylation of the $\mathrm{zc} 3 \mathrm{~h} / 2 \mathrm{~d}$ gene is significantly downregulated in LUAD.

positive correlation with $B$ cells $(r=0.325, p=1.30 \mathrm{e}-13)$, CD8 + T cells $(r=0.173, p=1.18 \mathrm{e}-04), \mathrm{CD} 4+\mathrm{T}$ cells $(\mathrm{r}=$ $0.399, \mathrm{p}=2.59 \mathrm{e}-20)$, neutrophils $(\mathrm{r}=0.343, \mathrm{p}=4.52 \mathrm{e}-15)$, dendritic cells $(\mathrm{r}=0.27, \mathrm{p}=1.13 \mathrm{e}-09)$, Tregs $(\mathrm{r}=0.117$, $\mathrm{p}=9.14 \mathrm{e}-03)$, and monocyte $(\mathrm{r}=0.204, \mathrm{p}=5.01 \mathrm{e}-06)$ immune infiltration levels of LUAD (Figure 6A).

To further validate these results, the relationships between zc3h12d expression and immune marker genes of different immune cells were explored by TIMER database, including B cells, CD8+ T cells, neutrophils, macrophages, dendritic cells, tregs, monocytes, NK cells, Th1 cells, Th2 cells, and Th17 cells in LUAD. Interestingly, we found that the levels of most immune markers were significantly correlations with zc3h12d expression in LUAD (Table 3).

As the tumor immune infiltration and immune marker of B cells exhibited significant correlations with zc $3 \mathrm{~h} 12 \mathrm{~d}$. 


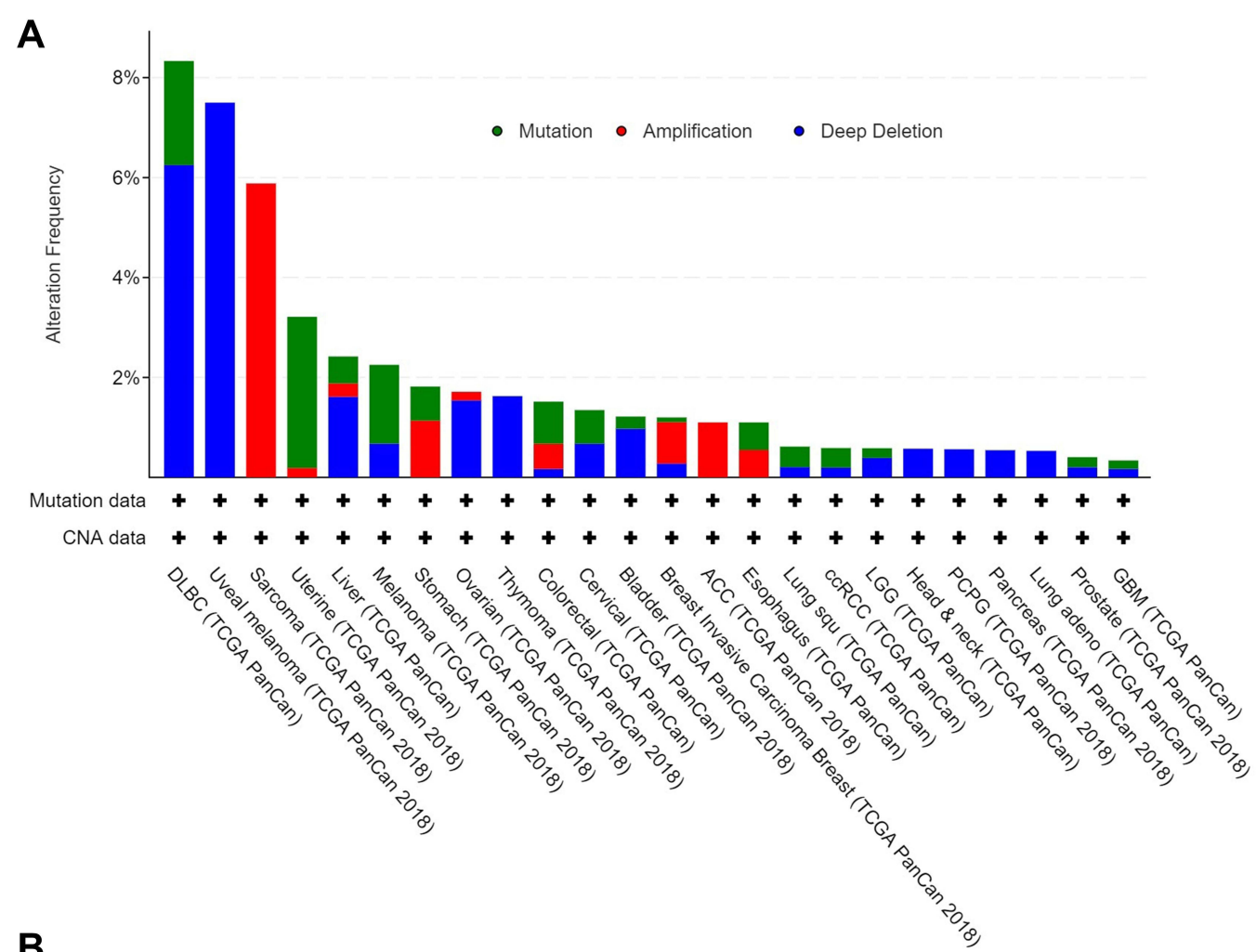

B
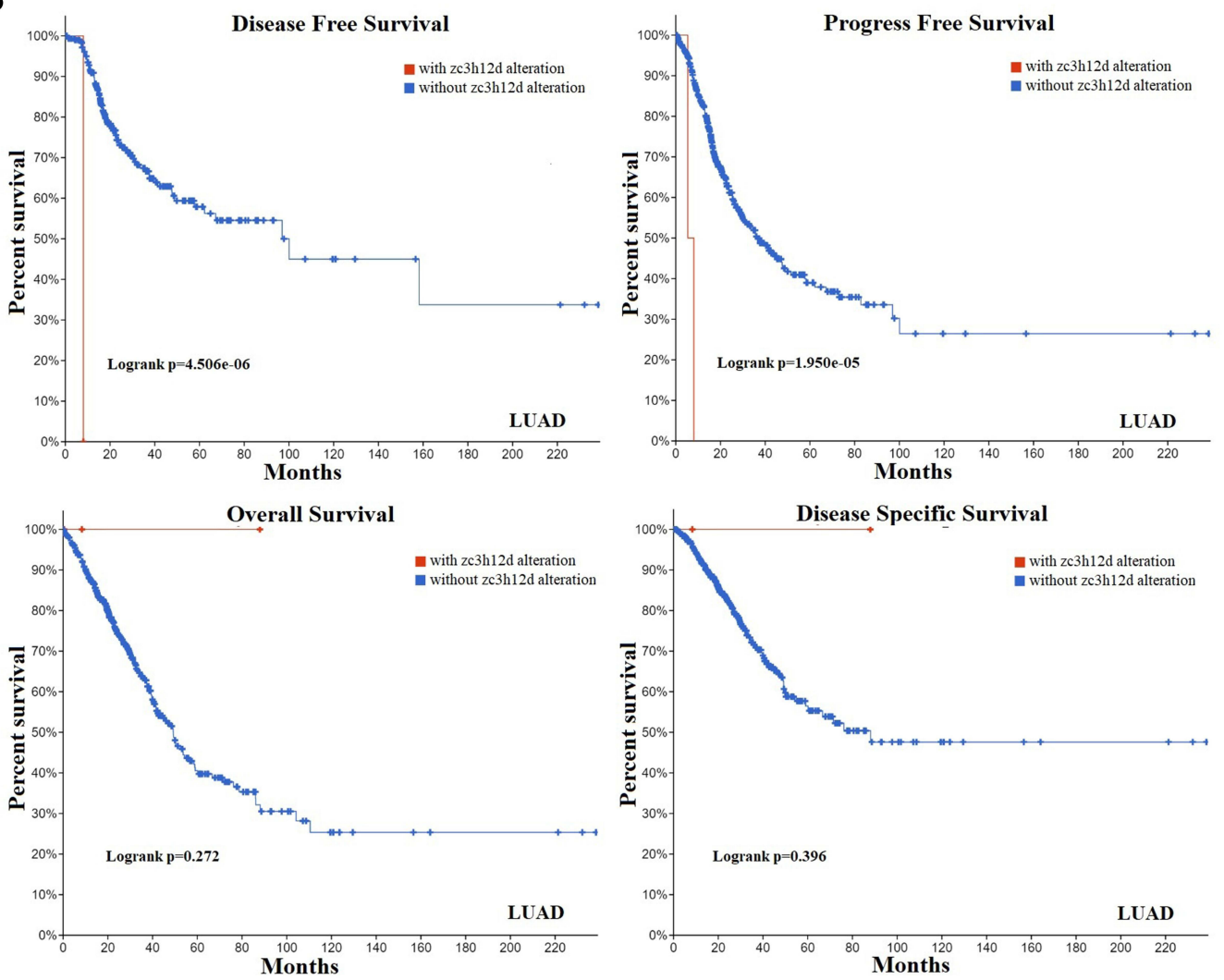

Figure 4 Gene alteration of zc3h $2 \mathrm{~d}$ in LUAD. (A) The alteration frequency of zc3h $2 \mathrm{~d}$ in different cancer types in the TCGA database. (B) K-M survival curves between $\mathrm{zc} 3 \mathrm{~h} / 2 \mathrm{~d}$ altered group and unaltered group by cBioportal. 

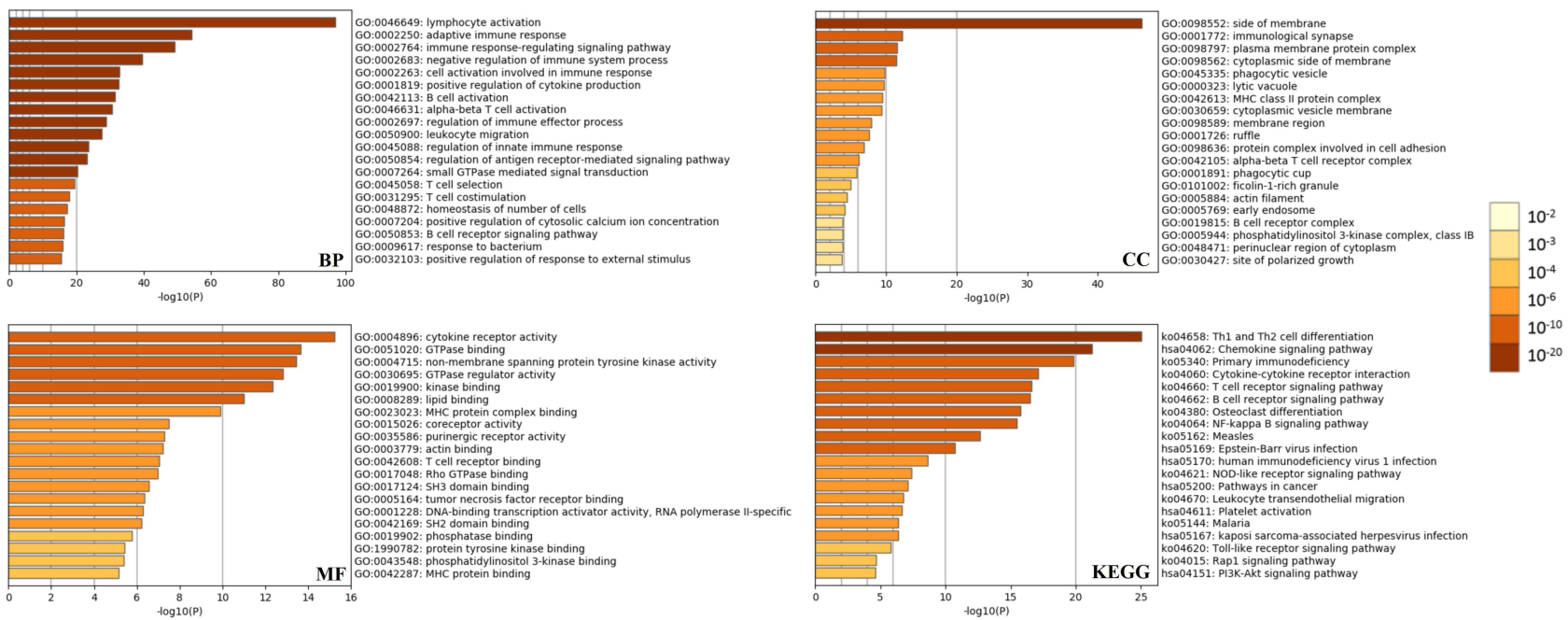

Figure 5 Functional enrichment of zc3h/2d. The gene ontology (GO) and Kyoto Encyclopedia of Genes and Genomes (KEGG) enriched terms colored according to P-values.

A

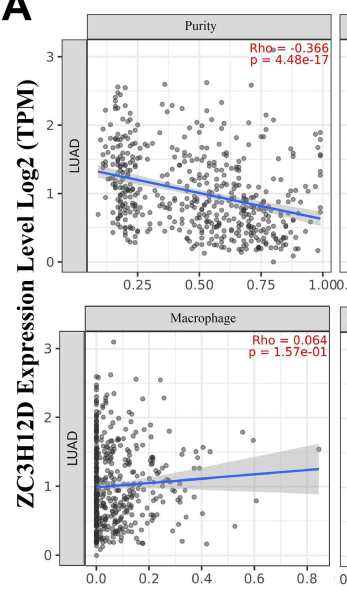

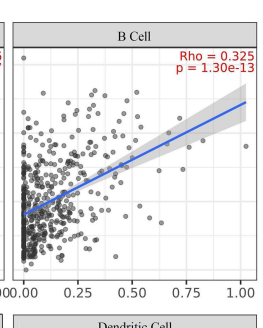

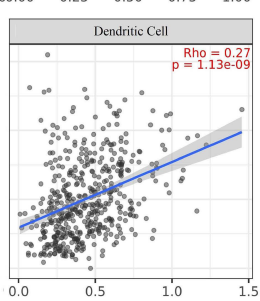

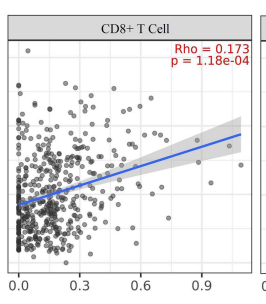
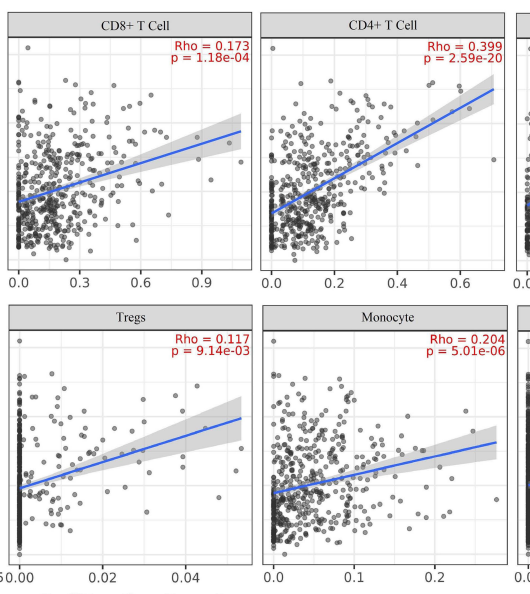
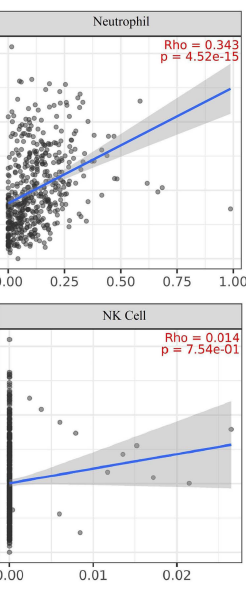

B

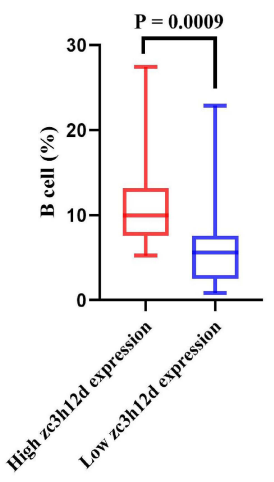

Figure 6 Correlation of $\mathrm{zc} 3 \mathrm{~h} / 2 \mathrm{~d}$ with immune infiltration level in LUAD. (A) The correlations between zc3h $12 \mathrm{~d}$ expression and infiltrating levels of immune cells, including $B$ cell $(p=1.30 e-13), C D 8+T$ cell $(p=1.18 e-04), C D 4+T$ cell $(p=2.59 e-20)$, neutrophil $(p=4.52 e-15)$, macrophage $(p=1.57 e-01)$, dendritic cells $(p=1.13 e-09)$, tregs $(p$ $=9.14 \mathrm{e}-03)$, monocyte $(p=5.0 \mathrm{le}-06)$, and NK cell $(p=7.54 \mathrm{e}-0 \mathrm{I})$ relative to $\mathrm{zc} 3 \mathrm{~h} / 2 \mathrm{~d}$ expression. $(\mathrm{B})$ Relations between the expression of $z c 3 \mathrm{~h} / 2 \mathrm{~d}$ and $B$ cells in LUAD $(p=$ $0.0009)$.

Then, B cells were selected for further study. The result confirmed that B cells level were significantly lower in patients with low zc3h12d expression than patients with high zc3h12d expression $(p=0.0009)$ (Figure 6B). These findings might provide an explanation for the differences in patient survival.

\section{Discussion}

To enhance the prediction of LUAD patients' prognosis, DNA methylation was demonstrated with vital prognostic value. ${ }^{18}$ DNA methylation on specific sites could regulate corresponding gene expression. ${ }^{19}$ In addition to the DNA methylation, the host immune response had emerged as a promising therapeutic option with the potential to improve OS in LUAD patients. ${ }^{20}$

Zc3h12a plays a critical role in $\mathrm{T}$ cell mediated immunosuppression. However, transformed follicular lymphoma (TFL, zc3h12d), an RNase belonging to the same family of Regnase-1 (zc3h12a), was originally reported as a putative tumor suppressor gene. ${ }^{21}$ Although zc3h12d had not been extensively studied, the anti-tumor properties were confirmed in cell experiments. Subsequently, the 
Table 3 Correlation Analysis Between zc3h I2d and Immune Cell Type Markers in TIMER Database

\begin{tabular}{|c|c|c|c|c|c|}
\hline \multirow[t]{2}{*}{ Cell Type } & \multirow[t]{2}{*}{ Gene Markers } & \multicolumn{2}{|c|}{ None } & \multicolumn{2}{|c|}{ Purity } \\
\hline & & COR & $\mathbf{P}$ & COR & $\mathbf{P}$ \\
\hline \multirow[t]{3}{*}{$B$ cell } & CDI9 & 0.607 & $3.92 e-53$ & 0.539 & $1.67 e-38$ \\
\hline & FCRL2 & 0.504 & $1.57 e-12$ & 0.422 & $9.25 e-23$ \\
\hline & MS4AI & 0.614 & $1.36 \mathrm{e}-54$ & 0.534 & I.IIe-37 \\
\hline \multirow[t]{2}{*}{ CD8+ T cell } & CD8A & 0.493 & $7.56 \mathrm{e}-33$ & 0.406 & $5.75 e-21$ \\
\hline & CD8B & 0.437 & 2. $19 \mathrm{e}-25$ & 0.364 & $7.37 e-17$ \\
\hline \multirow[t]{6}{*}{ Neutrophil } & CEACAM8 & 0.162 & $2.24 \mathrm{e}-04$ & 0.151 & $7.65 e-04$ \\
\hline & CCR7 & 0.68 & $2.94 \mathrm{e}-7 \mathrm{I}$ & 0.614 & $2.5 e-52$ \\
\hline & CSF3R & 0.351 & $2.13 e-16$ & 0.332 & $4.12 \mathrm{e}-14$ \\
\hline & FCGR3B & 0.236 & $5.8 \mathrm{e}-08$ & 0.172 & $1.27 e-04$ \\
\hline & FPRI & 0.384 & $1.65 e-19$ & 0.286 & $1.04 \mathrm{e}-10$ \\
\hline & SIGLEC5 & 0.515 & $3.28 e-36$ & 0.445 & $2.72 \mathrm{e}-25$ \\
\hline \multirow[t]{5}{*}{ Macrophage } & CD68 & 0.366 & $8.35 e-18$ & 0.274 & $6.54 \mathrm{e}-10$ \\
\hline & CD84 & 0.629 & $3.6 \mid \mathrm{le}-58$ & 0.562 & $2.47 e-42$ \\
\hline & CDI63 & 0.453 & I.78e-27 & 0.368 & $3.16 \mathrm{e}-17$ \\
\hline & IRF5 & 0.387 & $7.72 \mathrm{e}-20$ & 0.31 & $1.81 \mathrm{e}-12$ \\
\hline & VSIG4 & 0.329 & $1.66 \mathrm{e}-14$ & 0.243 & $4.55 e-08$ \\
\hline \multirow[t]{5}{*}{ Dendritic cell } & ITGAX & 0.589 & $1.94 \mathrm{e}-49$ & 0.525 & $3.15 e-36$ \\
\hline & CDIC & 0.344 & $1.02 \mathrm{e}-15$ & 0.258 & $6.18 \mathrm{e}-09$ \\
\hline & NRPI & 0.194 & $9.22 \mathrm{e}-06$ & 0.168 & $1.86 \mathrm{e}-04$ \\
\hline & HLA-DRA & 0.431 & $9.12 \mathrm{e}-25$ & 0.328 & $8.35 \mathrm{e}-14$ \\
\hline & HLA-DQBI & 0.374 & $1.47 e-18$ & 0.28 & $2.6 \mathrm{e}-10$ \\
\hline \multirow[t]{3}{*}{ Tregs } & STAT5B & 0.506 & 8.1Ie-35 & 0.515 & $8.62 e-35$ \\
\hline & FOXP3 & 0.619 & $7.85 e-56$ & 0.553 & $8.93 e-4 I$ \\
\hline & CCR8 & 0.637 & $6.9 e-60$ & 0.573 & $2.23 e-44$ \\
\hline \multirow[t]{3}{*}{ Monocyte } & CD86 & 0.512 & $1.03 e-35$ & 0.418 & $2.83 e-22$ \\
\hline & C3ARI & 0.489 & $2.7 \mathrm{Ie}-32$ & 0.401 & $1.62 \mathrm{e}-20$ \\
\hline & CSFIR & 0.521 & $3.91 \mathrm{e}-37$ & 0.438 & I.7e-24 \\
\hline \multirow[t]{4}{*}{ NK cell } & NCRI & 0.431 & $1.09 e-24$ & 0.37 & $2.08 \mathrm{e}-17$ \\
\hline & KIR2DLI & 0.264 & 1.2e-09 & 0.221 & $6.87 e-07$ \\
\hline & KIR2DS4 & 0.276 & $1.97 e-10$ & 0.226 & $3.83 e-07$ \\
\hline & KIR3DLI & 0.252 & $6.35 e-09$ & 0.2 & 7.47e-06 \\
\hline \multirow[t]{3}{*}{ ThI } & STATI & $0.34 I$ & $1.84 \mathrm{e}-15$ & 0.27 & $1.18 \mathrm{e}-09$ \\
\hline & TNF & 0.48 & $4.96 e-31$ & 0.385 & $7.16 \mathrm{e}-19$ \\
\hline & TBX2I & 0.576 & $8.7 e-47$ & 0.505 & $2.5 e-33$ \\
\hline \multirow[t]{3}{*}{ Th2 } & STAT5A & 0.59 & $1.28 \mathrm{e}-49$ & 0.518 & $3.06 \mathrm{e}-35$ \\
\hline & ILI3 & 0.202 & $3.63 e-06$ & 0.139 & $2.05 e-03$ \\
\hline & GATA3 & 0.399 & $4.35 e-21$ & 0.295 & $2.25 \mathrm{e}-1 \mathrm{I}$ \\
\hline Thl7 & ILI7A & 0.215 & $8.42 \mathrm{e}-07$ & 0.155 & $5.56 \mathrm{e}-04$ \\
\hline
\end{tabular}

Abbreviation: COR, correlation.

same role of zc3h12d in the $3^{\prime}$ UTRs was revealed, as zc3h12a. ${ }^{6}$ Simultaneously, the relationship between the primary human memory $\mathrm{T}$ lymphocytes and zc3h12d expression was reported as well. ${ }^{22}$
Even though the zc3h12d correlated with survival in endometrial cancer patients. ${ }^{21}$ Here, we report that $\mathrm{zc} 3 \mathrm{~h} 12 \mathrm{~d}$ may be considered as a potential biomarker for determining prognosis and immune-related therapeutic target in LUAD. 
High zc3h12d expression in LUAD is observed compared with adjacent normal tissues and immunohistochemistry shows that $\mathrm{zc} 3 \mathrm{~h} 12 \mathrm{~d}$ is mainly localized in cytoplasmic granules. Then, various clinic factors of zc3h12d expression are integrated in LUAD patients. Database analysis results show that $\mathrm{zc} 3 \mathrm{~h} 12 \mathrm{~d}$ expression is widely related with disease stage, ethnicity, gender, age, lymph node status, TP53 mutation status and smoking status in LUAD patients. However, sample detection of patients reveals that $\mathrm{zc} 3 \mathrm{~h} 12 \mathrm{~d}$ expression is closely related to pathological $\mathrm{N}(\mathrm{p}=0.0431)$ and grade $(\mathrm{p}=0.004)$. In comparison, other clinicopathological items, including age, gender, smoking status, $\mathrm{T}$ and TNM stage show that it has no statistical association with $\mathrm{zc} 3 \mathrm{~h} 12 \mathrm{~d}$ protein expression. We find low zc3h12d expression is closely related with poorer OS in LUAD. In addition, univariate and multivariate analyses indicates that lower expression of zc3h12d may be defined as a risk factor which affects the OS of LUAD patients.

Hypomethylation is one of the important components of epigenetic alterations of tumorigenesis and progression. ${ }^{23}$ Recent study has demonstrated that the promoter hypomethylation might be served as a prognostic indicators and drug target in lung cancer. ${ }^{24}$ In the course of our investigations, the promoter methylation levels of zc3h12d are significantly decreased. And the hypomethylation of zc3h12d may cause a significant increasing in $\mathrm{zc} 3 \mathrm{~h} 12 \mathrm{~d}$ expression.

It has been proved that genomic mutation is closely associated with tumorigenesis. ${ }^{25}$ Therefore, a genetic alteration analysis is conducted. Result indicates that genetic alteration of $\mathrm{zc} 3 \mathrm{~h} 12 \mathrm{~d}$ in NSCLC $(1.15 \%$ frequency) such as deep deletion, and mutation could be found. Intriguingly, all LUAD cases with genetic alteration ( $0.53 \%$ frequency) have copy number deletion of zc $3 \mathrm{~h} 12 \mathrm{~d}$. Considering all the above results, zc $3 \mathrm{~h} 12 \mathrm{~d}$ might play an important role in the disease progression and survival prognosis of patients with LUAD.

Similar with zc3h12a, zc3h12d is also a negative regulator of cytokine expression. In cell experiments, zc $3 \mathrm{~h} 12 \mathrm{~d}$ is proved as a novel negative feedback regulator of TLR signaling and macrophage activation. ${ }^{26}$ And zc3h $12 \mathrm{~d}$ recognizes the same 3'UTR-dependent regulation of the turnover of mRNAs encoding interleukin-6 (IL-6), tumor necrosis factor (TNF), and immediate early response 3 gene (IER3), as zc3h12a. ${ }^{6}$ The role of zc3h12d in pathogenic immune responses is also described. Recently, a strong correlation between zc3h12d expression and human $\mathrm{T}$ lymphocytes is reported. ${ }^{8}$ To further identify the mechanism of zc3h12d in LUAD, the zc3h12d-related gene set is obtained by employing UALCAN database. GO and KEGG pathway analysis results indicate that $\mathrm{zc} 3 \mathrm{~h} 12 \mathrm{~d}$ has a certain value in immune infiltration, such as the inhibition of cell proliferation in LUAD.

In the NSCLC tumor microenvironment (TME), the infiltrating immune cells account for a large proportion, including $\mathrm{T}$ cells, B cells, and macrophages, etc. ${ }^{27,28}$ In vivo-isolated human CD4+ T cells experiments, it is found that deficiency of $\mathrm{zc} 3 \mathrm{~h} 12 \mathrm{~d}$ could increase proinflammatory phenotype of T lymphocytes. ${ }^{8}$ In the present study, our data shows that there is a positive relationship between zc3h12d and infiltration level of B cells, CD8 + T cells, CD4 + T cells, neutrophils, dendritic cells, Tregs, and monocyte in LUAD. In addition, tumor-associated-B cells in NSCLC is related to a favorable outcome. ${ }^{29}$ Our results of patient sample detection confirm that B cells level is significantly lower in the patients with low zc3h12d expression than patients with high zc3h12d expression. As such, zc3h12d has significantly positively correlation with the immune cell-type markers in LUAD. Notably, markers of $\mathrm{T}$ helper cells, such as STAT1, TBX21, STAT5A, and IL17A also show positive association with zc3h12d. Some studies have revealed that tumor infiltration by Th1 CD4+ T cells, Th2 CD4+ T cells, and Th17 CD4+ $\mathrm{T}$ cells can mediate regression of advanced solid tumors. ${ }^{30}$ Thus, these evidences provide a potential mechanism of $\mathrm{zc} 3 \mathrm{~h} 12 \mathrm{~d}$ in tumor immune microenvironment for researchers.

\section{Conclusions}

In summary, there is a close correlation of $\mathrm{zc} 3 \mathrm{~h} 12 \mathrm{~d}$ with LUAD. Low zc3h12d expression is closely related with poorer prognosis in LUAD. Besides, zc3h12d may promote tumor-induced immune response activation and immune infiltration in LUAD and inhibit the proliferation of lung cancer to play an anticancer role. Although our results offer compelling evidence of zc3h12d in LUAD, further studies should be verified in multicenter, largesample in future.

\section{Declarations}

The authors declare no support from any organizations for the submitted work. The design of the study, the analyses and the writing of the manuscript were solely the responsibility of the authors. 


\section{Data Sharing Statement}

The data of the current research are available from the corresponding author on a reasonable request.

\section{Consent for Publication}

Not applicable

\section{Acknowledgments}

We thank Prof. Chang-Qing Sun, and Hui Wang from the department of Science and Education of Tianjin Baodi Hospital of Tianjin Medical University for their kind technical assistance. Bo Yang, Lin-Lin Ji and Hong-Liang Xu should be regarded as co-first authors.

\section{Disclosure}

The authors declare that there are no conflicts of interest.

\section{References}

1. Schneider BJ, Ismaila N, Aerts J, et al. Lung cancer surveillance after definitive curative-intent therapy: ASCO Guideline. J Clin Oncol. 2020;38(7):753-766. doi:10.1200/JCO.19.02748

2. Aizemaiti R, Wu Z, Tang J, et al. Heat shock factor 5 correlated with immune infiltration serves as a prognostic biomarker in lung adenocarcinoma. Int J Med Sci. 2021;18(2):448-458. doi:10.7150/ ijms.51297

3. Zhao W, Rong Z, Wang W, et al. Methylation biomarkers with discriminating ability are potential therapeutic targets in lung adenocarcinoma. Epigenomics. 2020. doi:10.2217/epi-2019-0142

4. Li Z, Ding B, Xu J, et al. Relevance of STK11 mutations regarding immune cell infiltration, drug sensitivity, and cellular processes in lung adenocarcinoma. Front Oncol. 2020;10:580027. doi:10.3389/ fonc. 2020.580027

5. Huang S, Qi D, Liang J, et al. The putative tumor suppressor Zc3h12d modulates toll-like receptor signaling in macrophages. Cell Signal. 2012;24(2):569-576. doi:10.1016/j.cellsig.2011.10.011

6. Wawro M, Kochan J, Krzanik S, et al. Intact NYN/PIN-Like domain is crucial for the degradation of inflammation-related transcripts by ZC3H12D. J Cell Biochem. 2017;118(3):487-498. doi:10.1002/ jcb. 25665

7. Zhang H, Wang WC, Chen JK, et al. ZC3H12D attenuated inflammation responses by reducing mRNA stability of proinflammatory genes. Mol Immunol. 2015;67(2Pt B):206-212. doi:10.1016/j. molimm.2015.05.018

8. Emming S, Bianchi N, Polletti S, et al. A molecular network regulating the proinflammatory phenotype of human memory $\mathrm{T}$ lymphocytes. Nat Immunol. 2020;21(4):388-399. doi:10.1038/ s41590-020-0622-8

9. Liang J, Wang J, Azfer A, et al. A novel CCCH-zinc finger protein family regulates proinflammatory activation of macrophages. $J$ Biol Chem. 2008;283(10):6337-6346. doi:10.1074/jbc.M707861200

10. Wang W, Zhang Z, Yang B, et al. [Down-regulation of Zc3h12d expression in lung tissues of rats with acute lung injury induced by intestinal ischemia-reperfusion]. Xi Bao Yu Fen Zi Mian Yi Xue Za Zhi. 2014;30(4):355-359.

11. Li T, Fan J, Wang B, et al. TIMER: a web server for comprehensive analysis of tumor-infiltrating immune cells. Cancer Res. 2017;77(21): e108-e110. doi:10.1158/0008-5472.CAN-17-0307
12. Chandrashekar DS, Bashel B, Balasubramanya SAH, et al. UALCAN: a portal for facilitating tumor subgroup gene expression and survival analyses. Neoplasia. 2017;19(8):649-658. doi:10.1016/j. neo.2017.05.002

13. Tang Z, Li C, Kang B, et al. GEPIA: a web server for cancer and normal gene expression profiling and interactive analyses. Nucleic Acids Res. 2017;45(W1):W98-W102. doi:10.1093/nar/gkx247

14. Győrffy B, Surowiak P, Budczies J, Lánczky A. Online survival analysis software to assess the prognostic value of biomarkers using transcriptomic data in non-small-cell lung cancer. PLoS One. 2013;8 (12):e82241. doi:10.1371/journal.pone.0082241

15. Modhukur V, Iljasenko T, Metsalu T, et al. MethSurv: a web tool to perform multivariable survival analysis using DNA methylation data. Epigenomics. 2018;10(3):277-288. doi:10.2217/epi-2017-0118

16. Gao J, Aksoy BA, Dogrusoz U, et al. Integrative analysis of complex cancer genomics and clinical profiles using the cBioPortal. Sci Signal. 2013;6(269):pl1. doi:10.1126/scisignal.2004088

17. Zhou Y, Zhou B, Pache L, et al. Metascape provides a biologist-oriented resource for the analysis of systems-level datasets. Nat Commun. 2019;10(1):1523. doi:10.1038/s41467-019-09234-6

18. Li R, Yang YE, Yin YH, et al. Methylation and transcriptome analysis reveal lung adenocarcinoma-specific diagnostic biomarkers. J Transl Med. 2019;17(1):324. doi:10.1186/s12967-019-2068-z

19. Koch A, Joosten SC, Feng Z, et al. Analysis of DNA methylation in cancer: location revisited. Nat Rev Clin Oncol. 2018;15(7):459-466. doi:10.1038/s41571-018-0004-4

20. García-González J, Ruiz-Bañobre J, Afonso-Afonso FJ, et al. PD-(L) 1 Inhibitors in combination with chemotherapy as first-line treatment for non-small-cell lung cancer: a pairwise meta-analysis. J Clin Med. 2020;9(7):2093. doi:10.3390/jcm9072093

21. Wakahashi S, Kawakami F, Wakahashi K, et al. Transformed Follicular Lymphoma (TFL) predicts outcome in advanced endometrial cancer. Cancer Epidemiol Biomarkers Prev. 2018;27 (8):963-969. doi:10.1158/1055-9965.EPI-17-0762

22. Minagawa K, Wakahashi K, Kawano H, et al. Posttranscriptional modulation of cytokine production in $\mathrm{T}$ cells for the regulation of excessive inflammation by TFL. J Immunol. 2014;192(4):1512-1524. doi:10.4049/jimmunol.1301619

23. Imperatori A, Sahnane N, Rotolo N, et al. LINE-1 hypomethylation is associated to specific clinico-pathological features in Stage I non-small cell lung cancer. Lung Cancer. 2017;108:83-89. doi:10.1016/j.lungcan.2017.03.003

24. Noguera-Uclés JF, Boyero L, Salinas A, et al. The Roles of Imprinted SLC22A18 and SLC22A18AS gene overexpression caused by promoter cpg island hypomethylation as diagnostic and prognostic biomarkers for non-small cell lung cancer patients. Cancers (Basel). 2020;12(8):2075. doi:10.3390/cancers12082075

25. Li Y, Yuan J. Role of deubiquitinating enzymes in DNA double-strand break repair. J Zhejiang Univ Sci B. 2021;22 (1):63-72. doi:10.1631/jzus.B2000309

26. Huang WQ, Yi KH, Li Z, et al. DNA methylation profiling reveals the change of inflammation-associated ZC3H12D in Leukoaraiosis. Front Aging Neurosci. 2018;10:143. doi:10.3389/fnagi.2018.00143

27. Sun J, Xie T, Jamal M, et al. CLEC3B as a potential diagnostic and prognostic biomarker in lung cancer and association with the immune microenvironment. Cancer Cell Int. 2020;20:106. doi:10.1186/s12935-020-01183-1

28. Liu F, Wu H. CC chemokine receptors in lung adenocarcinoma: the inflammation-related prognostic biomarkers and immunotherapeutic targets. J Inflamm Res. 2021;14:267-285. doi:10.2147/JIR.S278395

29. Yuan Q, Sun N, Zheng J, et al. Prognostic and immunological role of FUN14 domain containing 1 in pan-cancer: friend or foe? Front Oncol. 2020;10(9):1502. doi:10.3389/fonc.2019.01502

30. Davies SI, Barrett J, Wong S, et al. Robust production of merkel cell polyomavirus oncogene specific $\mathrm{T}$ cells from healthy donors for adoptive transfer. Front Immunol. 2020;11:592721. doi:10.3389/ fimmu.2020.592721 


\section{Publish your work in this journal}

The Journal of Inflammation Research is an international, peerreviewed open-access journal that welcomes laboratory and clinica findings on the molecular basis, cell biology and pharmacology of inflammation including original research, reviews, symposium reports, hypothesis formation and commentaries on: acute/chronic inflammation; mediators of inflammation; cellular processes; molecular mechanisms; pharmacology and novel anti-inflammatory drugs; clinical conditions involving inflammation. The manuscript management system is completely online and includes a very quick and fair peerreview system. Visit http://www.dovepress.com/testimonials.php to read real quotes from published authors.

Submit your manuscript here: https://www.dovepress.com/journal-of-inflammation-research-journal 